\title{
Correction to: Reply to the letter: $N$-Butyl Cyanoacrylate- Lipiodol Mixture for Endovascular Purpose: Polymerization Kinetics Differences Between In Vitro and In Vivo Experiments
}

\author{
Natsuko Hayashi $^{1,2} \cdot$ Yoshito Takeuchi $^{3}\left(\mathbb{D} \cdot\right.$ Hiroshi Miura $^{1} \cdot$ Yusuke Arima $^{4}$. \\ Mitsuaki Toda $^{5} \cdot$ Toshiyuki Okamoto $^{1} \cdot$ Shunsuke Asai $^{1} \cdot$ Koji Sakai $^{1}$ • \\ Tatsuya Hirota $^{1} \cdot$ Kei Yamada ${ }^{1}$
}

Published online: 2 October 2020

(C) Springer Science+Business Media, LLC, part of Springer Nature and the Cardiovascular and Interventional Radiological Society of Europe (CIRSE) 2020

\section{Correction to:}

Cardiovasc Intervent Radiol (2020)

43:1411-412

https://doi.org/10.1007/s00270-020-02599-y

We would like to correct one of the references that was listed incorrectly in our article:

Reference 1 was listed as "Hayashi N, Takeuchi Y, Miura $\mathrm{H}$, et al. Is the cessation of blood flow faster than the polymerization of an N-butyl cyanoacrylate- lipiodol

The original article can be found online at https://doi.org/10.1007/ s00270-020-02599-y.

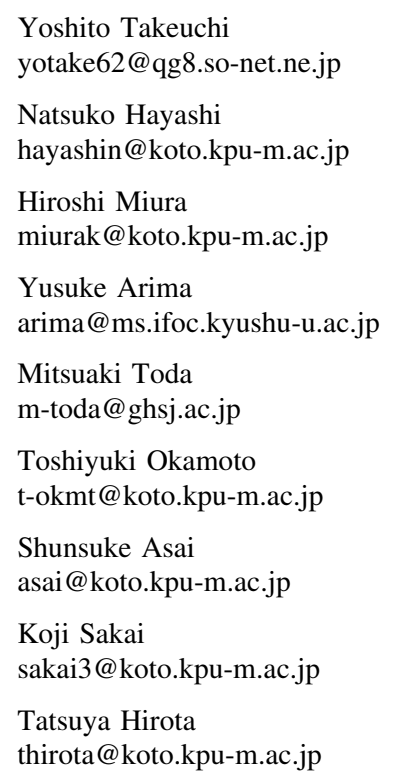

mixture? An in vitro phantom study. Cardiovasc Interv Radiol. 2020;43:630-5."

And it should be listed as "Loffroy R, Comby PO, Guillen K, et al. N-Butyl Cyanoacrylate-Lipiodol Mixture for Endovascular Purpose: Polymerization Kinetics Differences Between In Vitro and In Vivo Experiments. Cardiovasc Interv Radiol. 2020;43:1409-10.” instead.

Publisher's Note Springer Nature remains neutral with regard to jurisdictional claims in published maps and institutional affiliations.
Kei Yamada

kyamada@koto.kpu-m.ac.jp

1 Department of Radiology, Graduate School of Medical Science, Kyoto Prefectural University of Medicine, 465 Kajiicho, Kawaramachi Hirokoji, Kamigyo-ku, Kyoto 6028566, Japan

2 Present Address: Department of Radiology, Osaka General Hospital of West Japan Railway Company, 1-2-22 Matsuzakicho, Abeno-ku, Osaka 5450053, Japan

3 Department of Radiology, Fukuchiyama City Hospital, 231 Atsunakacho, Fukuchiyama, Kyoto 6208505, Japan

4 Institute for Materials Chemistry and Engineering, Kyusyu University, 744 Motooka, Nishi-ku, Fukuoka, CE 418190395, Japan

5 Graduate School of Health Care Sciences, Jikei Institute, 1-28 Miyahara, Yodogawa-ku, Osaka 5320003, Japan 